\title{
Comparison of various flooding procedures in reducing fear and in extinguishing jump-up avoidance responding
}

\author{
STEPHANIE MILLER, SUSAN MINEKA, and MICHAEL COOK \\ University of Wisconsin, Madison, Wisconsin
}

\begin{abstract}
Two experiments examined the effectiveness of three variations in flooding techniques on hastening extinction of a jump-up avoidance response (Experiment 1 ) and on reducing fear (Experiment 2) as assessed by the multivariate fear-assessment techniques of Corriveau and Smith (1978). Traditional flooding involved blocking the subject's response by making the safety ledge unavailable; barrier flooding involved inserting a Plexiglas barrier in front of the safety ledge to make it inaccessible and moving the wall periodically during treatment; no-barrier flooding involved allowing subjects to jump onto the ledge periodically but, if they did so, immediately dumping them back onto the grids. In both experiments, all three flooding treatments were found to be more effective than a home cage treatment, although the no-barrier procedure was significantly more effective than the other two. In addition, activity measures revealed interesting and significant group differences in the patterns of activity shown during treatment.
\end{abstract}

Over the years, a number of different variations in flooding or response-prevention techniques have been compared for their relative effectiveness in hastening the extinction of well-learned avoidance responses. The majority of studies have focused on comparisons of different techniques for flooding shuttlebox avoidance responses. With such responses, one can rather easily vary whether or not the response is allowed to occur, whether long or short CS exposures are used, and whether or not CS termination is response-contingent. Therefore, it is somewhat surprising that only a few studies have systematically varied these three factors and that, among those that have, there have been somewhat inconsistent results (e.g., Polin, 1959, vs. Berman \& Katzev, 1972). In a recent review of the literature, Mineka (1979) concluded that the bulk of the evidence suggested that, although the total amount of nonreinforced CS exposure was a very important factor, response prevention per se seems to facilitate shuttlebox avoidance response extinction, perhaps by allowing the learning of competing responses.

Comparing variations in flooding procedures for one-way jump-up avoidance responses, the primary focus of this article, is somewhat more complex than for shuttlebox responses because of differences in the nature of the CS and the response-CS termination contingency. In the jump-up apparatus there is

This research was supported by Grant BNS-7823612 from the National Science Foundation. We would like to thank Virginia Schloesser for her help in running these experiments. Requests for reprints should be sent to Susan Mineka, Department of Psychology, 1202 W. Johnson Street, University of Wisconsin, Madison, Wisconsin 53706. typically no discrete CS that is simply terminated by the response. Instead, the CS consists of the wall moving forward and dumping the rat onto the grid floor, followed by exposure to the grid floor and apparatus cues for some period of time before the shock comes on. An avoidance response is followed by CS termination only in the sense that the rat is allowed access to the safe ledge for some period of time; the rest of the CS (wall movement and the dumping process) is no longer even present when the response is made. During flooding, the typical procedure has been to make the ledge inaccessible and to force the rat to remain on the grid floor for a fixed period of time without being able to respond. Although this procedure has been found to be quite effective in hastening avoidance response extinction, it should be noted that it does not involve nonreinforced exposure to all parts of the CS during the flooding treatment. Specifically, the first and most salient part of the CS-wall movement and the dumping process-typically does not occur. In addition, this procedure does not allow the subject the opportunity to discover that when a response is made, it is no longer followed by access to the safe ledge for a period of time.

Perhaps because of the greater complexity of the $\mathrm{CS}$, and hence of the response-CS termination contingency, very little work has been directed to comparing the relative effectiveness of different flooding techniques in the jump-up box. In one experiment, Baum (1973) did find that a treatment that allowed the rat to respond but not to stay on the ledge (i.e., reducing the ITI to zero) was more effective in hastening avoidance response extinction than was a treat- 
ment that involved removing the ledge entirely. However, there are no published reports that have examined the effectiveness of simply exposing the rat to the wall-movement part of the CS without allowing it to respond. Thus, it is impossible to tell whether the superiority of Baum's technique, which allowed responding, stems from the additional CS exposure or from the rat's being allowed to respond per se.

The present experiments were designed, in part, to explore these issues more thoroughly by comparing the effectiveness, following jump-up avoidance training, of exposure to one of three different flooding techniques. In each experiment, one group of subjects received the traditional flooding treatment in which the wall was simply moved forward to make the ledge inaccessible for $5 \mathrm{~min}$. A second group also had the wall moved forward and a Plexiglas barrier inserted between the wall and the rest of the box. Once every $30 \mathrm{sec}$ for $6 \mathrm{~min}$, the wall went through a reverse cycle (forward-back-forward), thus exposing the rat to the sight and sound of the wall moving but still preventing responding. The third group also had the wall moved every $30 \mathrm{sec}$ in a reverse cycle, but no barrier was present and so the animals were allowed to respond at these times. However, if they got on the ledge, they were dumped off immediately by the wall's coming forward again. Thus, like the second group, they were exposed to the wall movement 10 times, but they were also exposed to the dumping process anywhere from 0-10 times, depending on how many times they responded.

Two further issues were also addressed in the present experiments. To date, none of the experiments that have systematically compared different flooding techniques have addressed the issue of the relative effectiveness of the various techniques in reducing fear of the CS. In the past, experiments that have examined the effectiveness of flooding in reducing fear have often found a considerable degree of dissociation between the effectiveness of a given amount of flooding in hastening avoidance response extinction and in reducing fear of the CS (e.g., Mineka \& Gino, 1979a; Mineka, Miller, Gino, \& Giencke, 1981). Research on variations of flooding techniques should also examine whether the same variables that are important for hastening avoidance response extinction are also important for reducing fear of the CS. Such research may be helpful in determining the mechanism underlying the effectiveness of these techniques and in determining how useful their human analogues may be in reducing different aspects of the fear response. Therefore, in the present experiments, we compared the effectiveness of three different flooding treatments not only in hastening jump-up avoidance extinction (Experiment 1), but also in reducing fear of the grid floor, using Corriveau and Smith's (1978) multivariate fear assessment techniques for the jump-up box (Experiment 2).
The third variable of interest in the present experiments concerned whether the behavior of the animals during the different flooding treatments would differ from each other and/or be predictive of the subsequent effect of the treatment on avoidance extinction or on fear. To date, little attention has been directed to this issue, in spite of the fact that the behavior exhibited during flooding may provide important clues regarding the mechanism(s) through which flooding exerts its effect. In one of the few studies that have addressed this issue, Baum (1969) reported that animals showing the most "relaxation" behavior (grooming and general activity) during treatment were the most likely to give up avoidance responding in extinction. However, there have been other suggestions that the freezing occurring during treatment may serve as an incompatible response that interferes with avoidance responding in extinction as well (e.g., Morokoff \& Timberlake, 1971). Therefore, the present experiments examined the behavior occurring during the three different treatments. The behavior of each rat was scored every $5 \mathrm{sec}$ as falling into one of six categories (freezing, grooming, abortive avoidance, rearing, locomotion, and general activity).

\section{EXPERIMENT 1}

\section{Subjects and Apparatus}

\section{Method}

The subjects were 64 male albino rats of the Fischer strain obtained from Harlan Sprague-Dawley Co., Madison, Wisconsin. The animals were 90-120 days of age at the time of the experiment. They were housed individually and maintained on ad-lib food and water throughout the experiment.

The apparatus consisted of an automated jump-up box obtained from Lafayette Company. In this apparatus, an $11.5 \times 20.0 \mathrm{~cm}$ section of one wall retracted, revealing a $12.5 \mathrm{~cm}$ high $\times 20.0 \mathrm{~cm}$ wide $\times 12.5 \mathrm{~cm}$ deep ledge $8.5 \mathrm{~cm}$ above a grid floor. The main chamber was constructed of Plexiglas (two walls and the lid) and sheet metal (the movable wall, ledge compartment, and wall opposite to the ledge). The floor consisted of 14 stainless steel grids with a $.48-\mathrm{cm}$-diameter placed $1.6 \mathrm{~cm}$ apart (center to center), to which a .7-mA constant current scrambled shock could be delivered. All observations and programming of experimental events were done in an adjacent room.

A $21.0 \times 20.0 \mathrm{~cm}$ removable Plexiglas barrier could be used to block subjects' attempts to reach the ledge. The carrying/retaining box was a plastic test cage, $29.0 \times 18.0 \times 13.0 \mathrm{~cm}$, with wood shavings on the bottom and a wire grid lid.

\section{Procedure}

The rats were assigned to four treatment groups of 16 subjects each. The experiment was divided into three phases over the course of 2 days: avoidance acquisition (Days 1 and 2), treatment (Day 2), and avoidance extinction (Day 2). In avoidance acquisition, each subject was placed on the ledge and was pushed off onto the grid floor $30 \mathrm{sec}$ later by the movement of the wall. Ten seconds later, the grid was electrified until the rat escaped by jumping or climbing onto the safety ledge, where it was allowed to remain for the $30-\mathrm{sec}$ ITI before being pushed off again, thus starting another trial. The subjects could avoid shock by jumping back onto the ledge within the 10-sec CS-US interval. Each subject was trained to a criterion of 10 consecutive avoidance responses on both Day 1 and Day 2. 
At the end of the ITI following the avoidance criterion on Day 2, all rats were removed from the apparatus and placed in the plastic carrying/retaining cage for $1 \mathrm{~min}$ before treatment began. The four groups received one of four different treatments for $5 \mathrm{~min}$ : (1) The home cage control group (HC) were returned to their home cages for $5 \mathrm{~min}$. (2) For subjects in the traditional flooding group (FL), the rear wall of the ledge compartment was moved to the forward position, making the safety ledge inaccessible to the rats. The subjects were placed on the grid floor and exposed to it for $5 \mathrm{~min}$ without the opportunity to make their usual avoidance response. (3) For subjects in the barrier flooding group (B), the clear Plexiglas barrier was inserted in front of the ledge and the wall was moved to the forward position. These subjects were placed on the grid floor for $5 \mathrm{~min}$, during which they were exposed to the wall moving on a reversed cycle (full cycle from front to back to front) once every $30 \mathrm{sec}$ without the opportunity to make avoidance responses. (4) Subjects in the no-barrier (NB) flooding group were treated identically to the $B$ animals, except that the barrier was not present. Thus, every $30 \mathrm{sec}$, when the wall moved to the backward position and revealed the safety ledge, the rat could make an avoidance response if it responded quickly. Each time an avoidance response was made, however, the rat was pushed back down to the grids within $2 \mathrm{sec}$, and it had to stay there another $30 \mathrm{sec}$. During the 5 -min treatment phase, there were 10 such opportunities for avoidance responding. No shocks were presented to any of the groups during this treatment phase.

During the treatment phase, observations from behind a oneway mirror were made of the subjects in the FL, B, and NB groups, using a time-sampling technique similar to that employed by Baum (1969). The experimenter observed and recorded, every $5 \mathrm{sec}$, the main activity of the rat (the activity taking the most time) during the preceding 5 -sec period. Thus, 60 observations were made during the $5 \mathrm{~min}$ of treatment. The rat's activity was divided into six categories: (1) abortive avoidance behavior-ledge-directed behaviors such as attempts to jump onto the retracted ledge, pushing against the ledge wall with the forepaws, and searching or sniffing around the ledge area; (2) freezing-remaining motionless on the grid floor without sniffing; (3) rearing-standing on the hindpaws with forepaws above the body midline; (4) locomotion-movements of the hindlegs through space; (5) groomingany licking, scratching, or rubbing of any part of the body; and (6) general activity-all behaviors that did not fit into one of the above categories.

At the conclusion of the 5-min treatment phase, all animals were put back in the plastic retaining box for $1 \mathrm{~min}$ while the apparatus was set up for avoidance extinction. During this phase, all rats were initially placed on the ledge by the experimenter and then pushed automatically onto the grid floor after the $30-\mathrm{sec}$ ITI, as in avoidance training. If a subject responded by jumping back onto the ledge, it could remain on the ledge for $30 \mathrm{sec}$, after which it was pushed back onto the grid floor. Responses that occurred within $10 \mathrm{sec}$ of being pushed onto the floor were scored as avoidance latency responses, while those that occurred after $10 \mathrm{sec}$ were scored as escape latency responses. No shocks were presented during this phase. If no responses were made, the wall continued to move every $30 \mathrm{sec}$. Extinction testing continued until an animal had made a total of 50 responses (escape or avoidance) or had failed to respond at all for $15 \mathrm{~min}$.

In all statistical analyses, the rejection level was set at $\mathrm{p}<.05$ unless otherwise indicated.

\section{Avoidance Acquisition}

\section{Results}

The number of shocks and trials needed to reach the criterion of avoidance acquisition on both Day 1 and Day 2 were analyzed in separate 4 (groups) $\times 2$ (repeated measures) ANOVAs. The analyses revealed only significant main effects of the repeated measure. The animals received significantly fewer shocks
$[F(1,60)=164.39]$ and took fewer trials $[F(1,60)=$ $77.42]$ to reach criterion on Day 2 than on Day 1 . The mean number of trials to criterion on Day 1 was 20.20 and on Day 2 was 12.17. The mean number of shocks on Day 1 was 5.27 and on Day 2 was 1.09.

\section{Activity Ratings}

Activity ratings were analyzed by breaking the 5-min treatment period into 1030 -sec blocks. Since observations were made every $5 \mathrm{sec}$, there were six observations that contributed to each 30 -sec block. All six activity ratings were then analyzed in separate 3 (treatment) $\times 10$ (repeated measures) ANOVAs. (The HC group did not have activity ratings made during the $\mathrm{HC}$ treatment.) The ANOVAs revealed significant treatment main effects for abortive avoidances $[F(2,45)=29.22]$, freezing $[F(2,45)=12.37]$, and grooming $[F(2,45)=10.22]$. Post hoc comparisons (Duncan, $\alpha=.05$ ) revealed that the NB group made more abortive avoidances than both the FL and $B$ groups, and that the FL group made more responses than the B group. The B group showed higher levels of freezing than the NB group, which, in turn, showed higher levels of freezing than the FL group. For grooming, the FL group showed higher levels than the B group, which, in turn, showed higher levels than the NB group. These results are illustrated in Figure 1.

There were also significant trial block main effects for abortive avoidances $[F(9,405)=22.70]$, freezing $[F(9,405)=6.00]$, and grooming $[F(9,405)=9.73]$, as well as significant treatment $\times$ trial block interactions for abortive avoidances $[\mathrm{F}(18,405)=4.37]$, freezing $[F(18,405)=3.14]$, and grooming $[F(18,405)=1.86]$. Analysis of simple main effects for grooming revealed that all three groups showed an increase in grooming across trial blocks $[\mathrm{Fs}(9,405) \geqslant 2.01]$. As can be seen in Figure 1, the FL group showed an earlier and greater

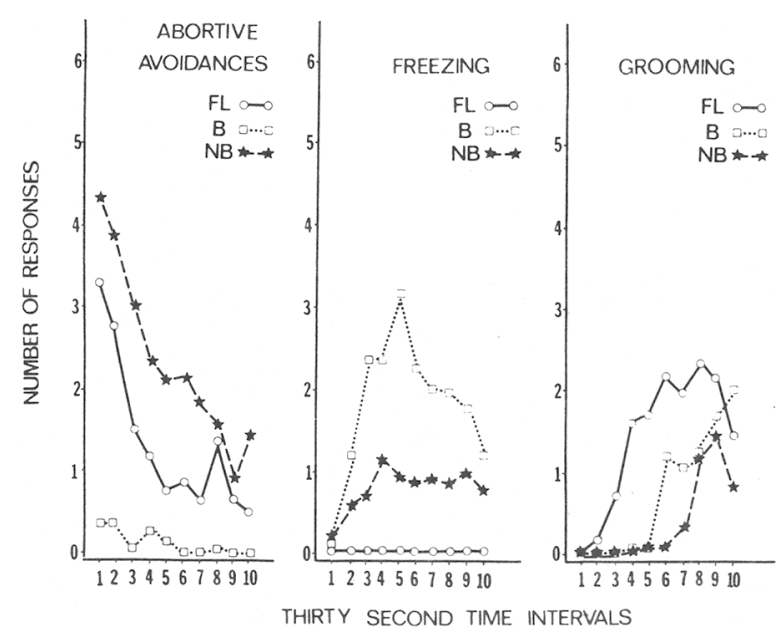

Figure 1. Mean number of 5-sec intervals in the 1030 -sec blocks of treatment that abortive avoidances, freezing, and grooming occurred for the FL, B, and NB groups of Experiment 1. 
increase than the other two groups. Analysis of the simple main effects for freezing revealed that the B group showed changes (first an increase and then a decrease) in levels of freezing across trial blocks $[F(9,405)=11.16]$. The $F L$ and NB groups did not show changes in freezing across trial blocks (Fs $\leqslant$ 1.12). (The FL group showed no freezing at all.) Analysis of the simple main effects for abortive avoidances revealed that both the FL and NB groups showed decreases across trial blocks $[\mathrm{Fs}(9,405)=10.5]$. The B group showed very low levels of abortive avoidance across all trial blocks. The other categories of activity (rearing, locomotion, and other) did not show any interesting effects.

\section{Avoidance Extinction}

The number of avoidance latency responses made in extinction were counted for each of 5 blocks of 10 trials. If a subject stopped responding completely, zeros were given for the remainder of the 50 trials. These data were then analyzed in a 4 (treatment) $\times 5$ (repeated measures) ANOVA. The ANOVA revealed only a significant main effect for treatment $[F(3,60)$ $=4.79$ ]. Post hoc comparisons (Duncan, $\alpha=.05$ ) revealed that the $\mathrm{HC}$ group responded more than each of the other three groups, and that the FL and B groups both responded more than the NB group. These results are shown in Figure 2.

Correlations were computed to determine whether there were any relationships between the various activities during treatment and the number of avoidance responses subsequently made in extinction. Abortive avoidances, freezing, and grooming were each correlated with avoidance responses, separately

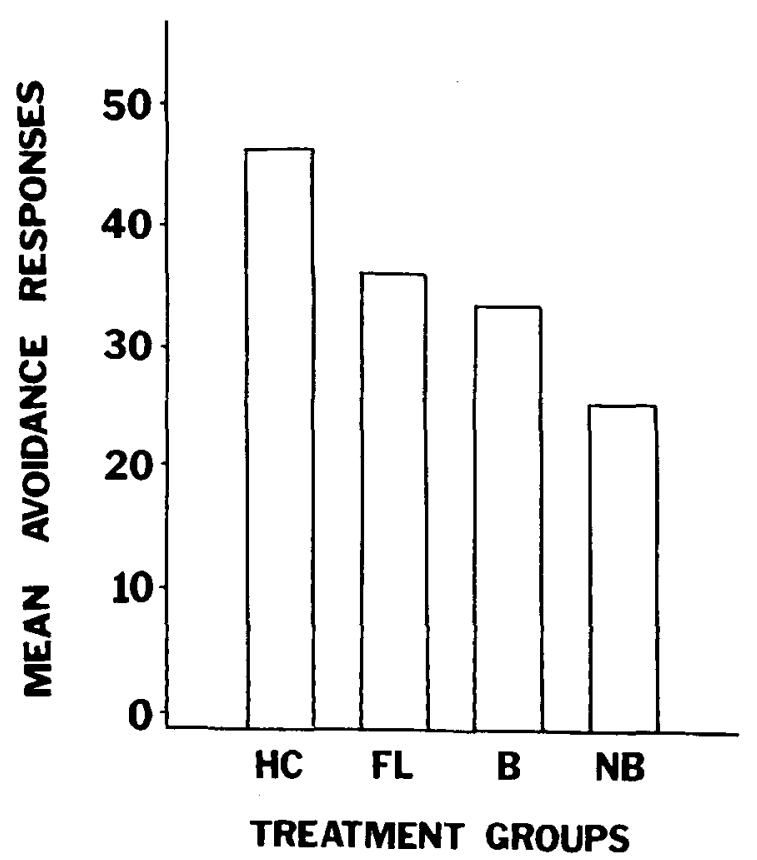

Figure 2. Mean number of avoidance responses made in extinction by the HC, FL, B, and NB groups of Experiment 1. for each group and collapsing across all three treatment groups. The only significant correlation was between number of avoidance responses and freezing for the B group $(r=.61)$, indicating that the rats who froze the most during treatment tended to make the most responses in extinction.

\section{Discussion}

The results of Experiment 1 indicate that although all three forms of flooding treatment were effective in hastening avoidance response extinction, the NB treatment was more effective than the other two. In other words, the animals that were allowed to respond but not to remain on the ledge subsequently showed the fewest avoidance responses in extinction. These results are consistent with those of Baum (1973), who also found a similar treatment to be more effective than a conventional flooding treatment. Since the NB treatment was superior to the B treatment, it seems that exposure to the wall movement per se is not sufficient to produce enhanced efficacy of flooding. Instead, some aspect of the dumping process (another part of the CS) and/or the elimination of the response-CS termination contingency seems to be more important for enhancing the effect of flooding. Unfortunately, strong conclusions about the unique importance of these variables cannot be made at the present time. Baum (1973), who used a different version of the jump-up apparatus, also found another treatment, involving placing a barrier parallel to the grid floor at the level of the ledge (thereby making the ledge inaccessible and allowing minimal movement on the part of the rat), to be as effective as his equivalent of the NB treatment. Thus, it is possible that there are several different optimal procedures, with each one perhaps producing its effect through a different mechanism.

One possible interpretation of the superiority of the NB procedure, other than the added nonreinforced CS exposure and/or the elimination of the response-CS termination contingency, stems from the possibility that the process of immediately being dumped onto the grids after jumping onto the ledge serves as a mild punishment for the response. By this analysis, the NB group responded less in extinction because their responses had been punished during treatment. Such a hypothesis should predict that the animals who made the most responses during treatment would have made the fewest avoidance responses in extinction. However, the correlation between number of responses during treatment $(0-10)$ and the number during extinction (0-50) was not significant $(r=.13)$. Thus, the punishment analysis of these results does not seem plausible. Instead, it seems more likely that the extra nonreinforced CS exposure and/ or elimination of the response-CS termination contingency were the critical variables that resulted in the superiority of the NB treatment.

Two other interesting aspects of our results stem 
from analysis of the activity ratings during treatment. First, there were clear-cut group differences in which activities tended to predominate. Not surprisingly, the NB group, which was allowed to make occasional real responses, also made the most abortive avoidance responses. And the FL group, which did not have a barrier present in front of the ledge and wall, made more abortive avoidance responses than the $B$ group. It is also of interest that the B group showed the highest levels of freezing and the FL group showed the lowest, and that the FL group showed the highest levels of grooming and the NB group showed the lowest. These latter results are of special interest because they suggest that Baum's $(1969,1970)$ earlier conclusions regarding the importance of the emergence of "relaxation" behaviors (grooming and general activity) before flooding will be effective may have been overstated. Comparing across groups, we found that the group showing the least grooming (NB) also made the fewest ARs in extinction, and the group showing the most grooming (FL) made the greatest number of ARs in extinction. Furthermore, there were no significant correlations between grooming (even combined with the other general activity measures) and the number of avoidance responses in extinction. Thus, our results do not support the contention of Baum regarding the importance of the emergence of relaxation behaviors for flooding to be effective.

The second interesting aspect of our activity results concerns the pattern of changes in activity across the 5-min treatment periods. Here our results tend to confirm those of Baum (although there were some group differences). In general, abortive avoidances decreased across the 5-min period, while grooming showed a substantial increase in all three groups. Freezing first showed an increase followed by a decrease or leveling off (except for the FL group, which did not freeze). Thus, although we did not find these activity measures to be significantly related to the treatment outcome, we did find a similar pattern of changes in activity to that found by Baum.

As stated in the introduction, there have not yet been any published reports of experiments that have systematically examined variations in flooding procedures with regard to their ability to reduce fear of the CS. Yet such results are of importance for several reasons. First, they would allow for the assessment of whether or not it is plausible to hypothesize that fear reduction may be involved in mediating the effects of flooding on hastening avoidance extinction. Second, such results may be of interest to human behavior therapy researchers who are interested in enhancing the effectiveness of their techniques, not only for reducing behavioral avoidance, but also for reducing other signs of fear and distress. Therefore, in Experiment 2, we examined the effectiveness of these same three techniques in reducing fear of the grids rather than in hastening AR extinction. Fear was assessed with Corriveau and Smith's (1978) multivar- iate technique, which measures the rat's willingness to safety test and approach the grid floor, as well as the amount of time spent on the grid floor. Although this technique allows assessment only of fear of the grid floor components of the CS, no other techniques that allow assessment of fear of the wall movement and dumping components of the CS are currently available.

The one major difference between our fear observation procedure and that of Corriveau and Smith was that our animals were dumped once off the ledge at the outset of the observation procedure and their's were not. This was done for two reasons. First, it maximized the similarity to Experiment 1, in which avoidance extinction also commences with the dumping procedure and thus with one exposure to the complete CS. Second, previous research in our laboratory had shown this variant on their fear observation procedure to be somewhat more sensitive in detecting flooding treatment effects when small amounts of flooding were used (see Mineka et al., 1981).

\section{EXPERIMENT 2}

\section{Subjects and Apparatus}

\section{Method}

The subjects were 72 naive male Fischer rats obtained from Harlan Sprague-Dawley Co., Madison, Wisconsin. The animals were 90-120 days old at the time of the experiment. Housing conditions were identical to those in Experiment 1. The apparatus was also identical to that described in Experiment 1.

\section{Procedure}

The avoidance training, treatment phase, and activity ratings ${ }^{1}$ were conducted in the same manner as in Experiment 1. In Experiment 2, however, instead of assessing avoidance response strength following treatment, the treatment phase was followed by a 1-h assessment of the subjects' fear of the grid floor using the observational techniques developed by Corriveau and Smith (1978). Following the 1-min retention interval, the subjects were placed on the safety ledge and allowed to remain there for $30 \mathrm{sec}$ before being pushed off onto the grid floor. The 60 -min fear-assessment phase began at this point, and if the subject jumped back onto the ledge, no further dumping occurred. During the fear assessment phase, nine dependent variables were used for the four groups:

First time on the grids after dumping (jump latency)-the latency of return to the ledge. The time for the subsequent dependent variables was counted from the point of return to the ledge. (This measure was not used by Corriveau and Smith, because their subjects were not dumped at the start of the fear observation phase.)

Approach latency - the time in seconds it initially took the subject to leave the ledge voluntarily.

Safety-test latency-the time it took the subject to make the initial safety test (1-2 paws or nose touching the grid floor). If the subject approached the grid floor without a safety test, this score was identical to the approach latency score.

Number of safety tests before first approach-the total number of safety tests that occurred before the first complete departure from the ledge.

Total number of safety tests-the total number of safety tests that occurred over the course of the 60 -min session. (This measure was not used by Corriveau and Smith.)

Total number of approaches-the number of times the subject voluntarily went back and forth from the ledge to the grids. The first dumping was not counted as an approach. 
Time initially spent on the grids after the first approach-the total amount of time in seconds spent on the grids after the first approach and before the first return to the ledge (recorded because all except five of the subjects that approached the grids at least once voluntarily also returned to the ledge at least once after the first approach). The subjects that never left the ledge received a 0 for this measure, and the five subjects that never returned to the ledge received a score equal to the remaining seconds in the session $(3,600$ minus the approach latency score plus the jump latency score).

Total voluntary grid time-the total cumulative number of seconds during the 60-min assessment period that the animal spent on the grids after the first approach.

Total grid time plus jump latency - an additional measure that combined the two scores for jump latency and total grid time (used because, since subjects spent time on the grids after being dumped before their first approach, their total grid time scores-see abovewere not completely representative of the total amount of time they did spend on the grids).

All these dependent variables were scored by hand by the experimenter, who observed the rat in the jump-up box from behind a one-way mirror in the control room. Periodic reliability tests were made with a second observer present. The interrater reliability checks revealed the intraclass correlations to be over .993 for each of the nine dependent variables $($ mean $=.997$ ) (cf. Tinsley $\&$ Weiss, 1975).

\section{Avoidance Acquisition}

\section{Results}

The number of shocks and trials to reach criterion on both Day 1 and Day 2 were analyzed in separate 4 (groups) $\times 2$ (repeated measures) ANOVAs. The analyses revealed only significant main effects of the repeated measure. The animals received significantly fewer shocks $[F(1,68)=78.58]$ and took fewer trials $[F(1,68)=41.68]$ to reach criterion on Day 2 than on Day 1. The mean number of trials to criterion on Day 1 was 18.66 and on Day 2, 13.33. The mean number of shocks on Day 1 was 4.48 and on Day 2 was 1.19 .

\section{Activity Ratings}

All six activity ratings were analyzed in separate 3 (treatment) $\times 10$ (repeated measures) ANOVAs. The ANOVAs revealed significant treatment main effects for abortive avoidances $[F(2,27)=6.29]$, freezing $[F(2,27)=3.81]$, and rearing $[F(2,27)=5.63]$. Post hoc comparisons (Duncan, $\alpha=.05$ ) revealed that the NB group made more abortive avoidances than both the FL and B groups and that the FL group made more responses than the B group. The B group showed higher levels of freezing than both the NB and FL groups, which did not differ significantly. The FL group showed higher levels of rearing than did both the $B$ and NB groups. These results are illustrated in Figure 3.

There were also significant trial blocks main effects for abortive avoidances $[F(9,243)=14.32]$ and grooming $[F(9,243)=10.92]$, as well as significant treatment $\times$ trial block interactions for abortive avoidances $[F(18,243)=3.03]$ and freezing $[F(18,243)$ $=1.63]$. The trial blocks effect for grooming was the result of an increase for all groups across trial blocks. Analysis of the simple main effects for abortive avoid-

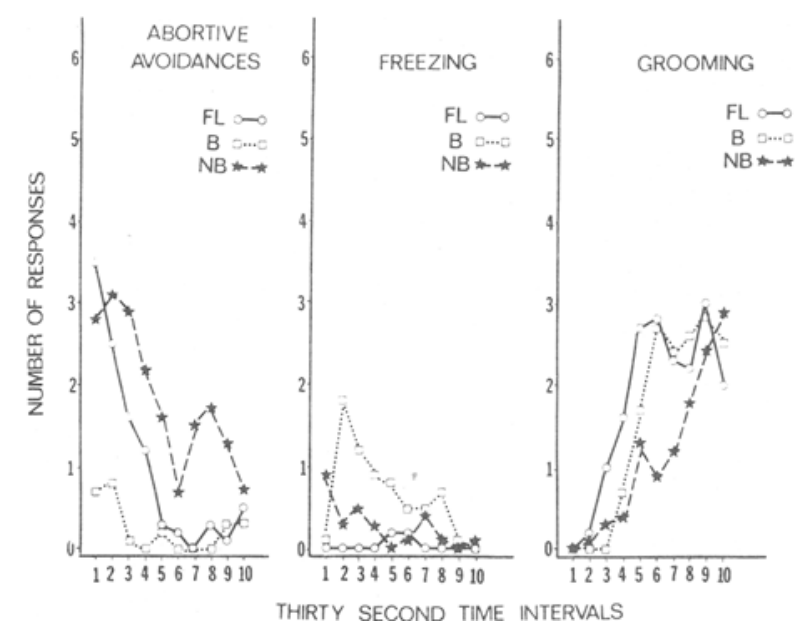

Figure 3. Mean number of 5-sec intervals in the 10 30-sec blocks of treatment that abortive avoidances, freezing, and grooming occurred for the FL, B, and NB groups of Experiment 2.

ances revealed that both the FL and the NB groups showed decreases across trial blocks $[\mathrm{Fs}(9,243) \geqslant 5.6]$. The B group showed very low levels of abortive avoidance across all trial blocks. Analysis of the simple main effects for freezing showed that the B group showed changes in levels of freezing (first an increase and then a decrease) across trial blocks $[F(9,243)=$ 3.78]. (The other two groups showed very low levels of freezing across all trial blocks.) The other categories of activity (locomotion and other) did not show any interesting effects.

\section{Fear Observations}

Inspection of the data revealed mild to severe heterogeneity of variance for seven of the fear measures (except for safety and approach latency). In addition, all measures had distributions that were severely skewed and clearly not normal in the tails. Therefore, for all measures the data were analyzed with nonparametric statistics. All of the nine fear measures were intercorrelated, as was done by Corriveau and Smith (1978) and Mineka et al. (1981). The Kendall rank correlations collapsing across all four groups are reported in Table 1. As may be seen in this table, there were many very high correlations of the different fear measures with each other. In particular, seven of the measures showed especially strong relationships. For example, number of approaches was negatively correlated with safety $(T=-.61)$ and approach $(\mathrm{T}=-.64)$ latencies, and positively correlated with first $(T=.72)$ and total $(T=.79)$ grid times, jump latency $(T=.32)$, and total grid time plus jump latency $(T=.60)$. It should be noted that, since some of the indices are not wholly independent of each other because of constraints imposed by the fear measurement techniques, some of the high correlations are not very meaningful. For example, safety and ap- 
Table 1

Kendall Rank Correlation Coefficients Between Each Pair of Variables for All 72 Subjects of Experiment 2

\begin{tabular}{lccccccrcr}
\hline & SL & AL & SFT & TST & NA & FG & TG & TG+JL \\
\hline Safety Latency (SL) & & & & & & & & \\
Approach Latency (AL) & .92 & & & & & & & \\
Safety Tests to First Approach (SFT) & -.17 & -.07 & & & & & & \\
Total Safety Tests (TST) & .33 & -.24 & .80 & & & & & \\
Number of Approaches (NA) & -.61 & -.64 & .14 & .32 & & & & \\
First Grid Time (FG) & -.68 & -.70 & .04 & .13 & .72 & & & \\
Total Grid Time (TG) & -.70 & -.73 & .04 & .17 & .79 & .92 & & .77 \\
Total Grid Time Plus Jump Latency (TG+JL) & -.70 & -.74 & .03 & .13 & .60 & .73 & .77 & .68 \\
Jump Latency (JL) & -.43 & -.46 & .01 & .01 & .32 & .41 & .38 \\
\hline
\end{tabular}

Note-When $T \geqslant .158, p<.05$, for all measures except jump latency. For correlations of jump latency with the other eight variables, $n=68$ because 4 subjects had no jump latency. For these correlations, when $T \geqslant .163, p<.05$.

proach latencies were sometimes identical, as were first and total grid times. In addition, if a subject had a very long approach latency, it could not have a long grid time (although short approach latencies were not necessarily followed by long grid times).

It should also be noted that safety tests to the first approach were significantly correlated only with total safety tests $(T=.80)$. Total safety tests were significantly correlated with three signs of a relative lack of fear (approach latency, $\mathrm{T}=-.24$; total grid time, $T=.17$; and number of approaches, $T=.32$ ), but these correlations were not of as great a magnitude as the others discussed above.

Correlations among the fear measures were also computed for each of the three treatment groups separately. The patterns of significance were nearly identical to those in Table 1 except that many of the correlations of other measures with jump latency, and with the two safety test measures were not significant when each group was looked at alone.

\section{Jump Latency}

Three subjects from the NB group and one subject from the FL group came down from the ledge before being dumped and therefore had no jump latency. For the remaining 68 subjects, the latency to return to the ledge after being dumped onto the grid floor was analyzed with a Kruskal-Wallis one-way analysis of variance which revealed that there were significant group differences $(H=34.59)$. Subsequent MannWhitney $U$ tests revealed that the FL, B, and NB groups all took longer to return to the ledge than did the $\mathrm{HC}$ group (Us $=85.5,42.5$, and 7.5, respectively). In addition, the NB group took longer to return than the FL and B groups (Us $=44.5$ and 49 , respectively). These results are illustrated in Figure 4.

\section{Safety and Approach Latency}

Because of the very high correlation between the measures of safety and approach latency, and because of the very similar pattern of treatment effects, the results of the analysis of these two measures are presented together. Kruskal-Wallis one-way analyses of variance revealed that there were significant group differences on each measure $(\mathrm{Hs}=16.55$ and 16.17 for safety and approach latencies, respectively). Subsequent Mann-Whitney $U$ tests revealed that the FL, $B$, and NB groups all safety tested sooner than the HC group (Us $=70,66.5$, and 32 , respectively). In addition, the NB group safety tested sooner than the B group $(U=98)$. Mann-Whitney $U$ tests also revealed that the FL, B, and NB groups approached the grids sooner than the HC group (Us =62.5, 54, and 29.5, respectively). The NB group also approached the grids sooner than the $B$ group $(U=97)$. These results are illustrated in Figure 5.

\section{Safety Tests to the First Approach and Total Safety Tests}

Because of the high correlation between safety tests to the first approach and total safety tests, and because of the similar results, these two measures are

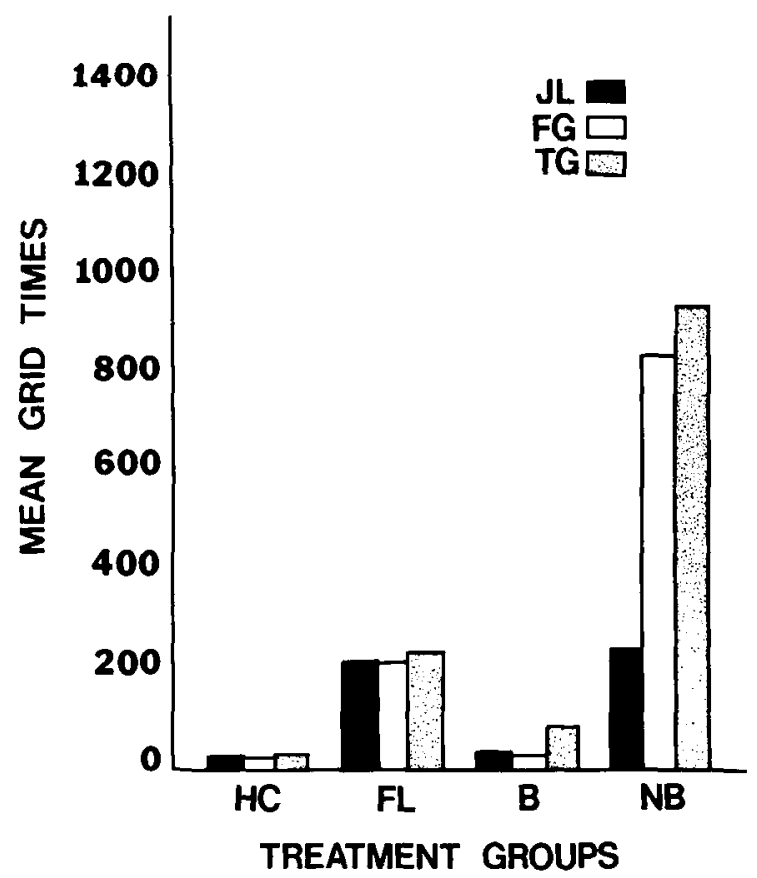

Figure 4. Mean jump latency (JL), first grid time (FG), and total grid time (TG) in seconds for the HC, FL, B, and NB groups of Experiment 2. 


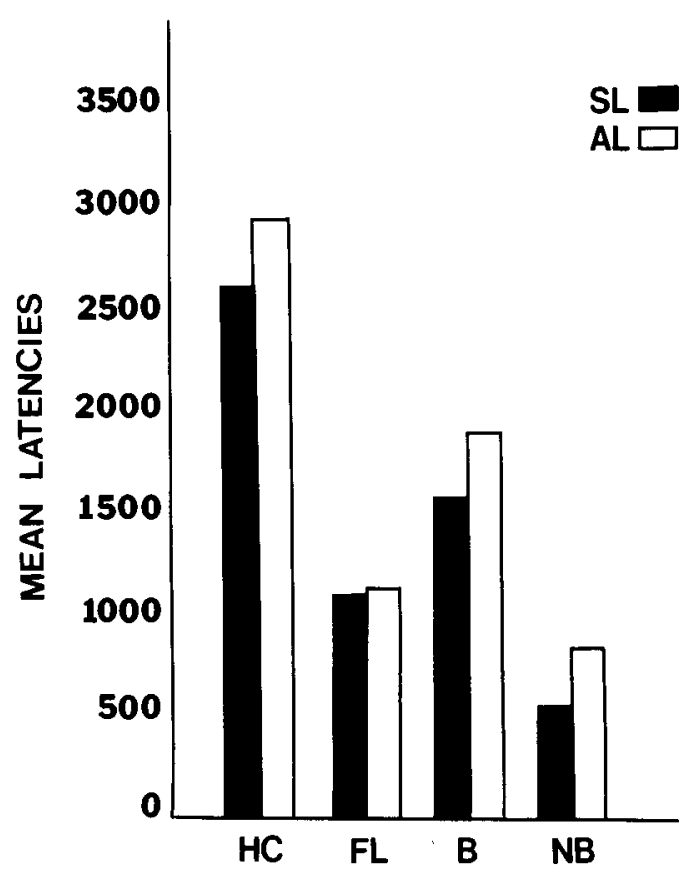

\section{TREATMENT GROUPS}

Figure 5. Mean safety latency (SL) and approach latency (AL) in seconds for the HC, FL, B, and NB groups of Experiment 2.

presented together. Kruskal-Wallis one-way analyses of variance revealed that there were no significant group differences for either measure $(\mathrm{Hs}=1.87$ and 2.40 , respectively). The mean numbers of safety tests to the first approach were $.39, .61, .56, .50$ for the FL, B, NB, and HC groups, respectively. The mean numbers of total safety tests were $.78,1.33,1.33, .50$ for the FL, B, NB, and $\mathrm{HC}$ groups, respectively.

\section{Number of Approaches}

A Kruskal-Wallis one-way analysis of variance revealed that there were significant group differences $(H=15.25)$ in number of approaches. Subsequent Mann-Whitney U tests revealed that both the FL and the NB groups made significantly more approaches to the grids than did the HC group (Us $=77$ and 50, respectively). The $B$ group differed only marginally from the HC group $(U=105.5)$. The mean number of approaches were $1.78,2.72,3.28, .33$ for the FL, $\mathrm{B}, \mathrm{NB}$, and $\mathrm{HC}$ groups, respectively.

\section{First and Total Grid Times}

Because of the very high correlation between first and total grid times and because of the very similar patterns of treatment effects for these measures, the results of first and total grid times are presented together. In fact, four subjects in the NB group and one subject in the FL group never came up after the first approach, and so their first and total grid times were identical. Kruskal-Wallis one-way analyses of variance revealed that there were significant group differences for each measure $(\mathrm{Hs}=21.36$ and 20.15 for first and total grid times, respectively). Subsequent Mann-Whitney $U$ tests revealed that the FL and NB groups spent more time on the grids than the $\mathrm{HC}$ group, both the first time and overall (Us $=73.5$ and 35 for FL and NB for first grid time, respectively, and Us $=75$ and 37 for FL and NB for total grid time, respectively). The B group spent only marginally longer time on the grids than the $\mathrm{HC}$ group (Us $=109$ and 107 for the first and total grid time, respectively). In addition, the NB group spent more time on the grids than both the FL and the B groups (Us $=79.5$ and 68.5 for FL and B groups for first grid time, and Us $=87.5$ and 74.5 for total grid time for FL and B groups, respectively). These results are illustrated in Figure 4.

\section{Total Grid Time Plus Jump Latency}

This measure, which reflects both voluntary and involuntary grid time, also showed significant group differences $(H=32.57)$. The $F L, B$, and NB groups all spent more time on the grids than the $\mathrm{HC}$ group (Us $=60.5,42$, and 14.5 , respectively). In addition, the NB group spent more time on the grids than the FL and B groups (Us $=74.5$ and 55, respectively).

\section{Correlations of Activity Measures with \\ Fear Observation Measures}

Kendall rank-order correlations were computed in order to determine whether there were any relationships between the various activities during treatment and subsequent levels of fear shown during the 60-min observation period. Abortive avoidances, freezing, and grooming (the only activities to show interesting group differences or changes over trial blocks) were each correlated with all nine fear measures, separately for each of the three treatment groups and collapsing across all three groups.

When all three groups were considered together, it was found that grooming during treatment was significantly correlated with safety latency $(\mathrm{T}=-.28)$, approach latency $(T=-.33)$, first grid time $(T=.27)$, total grid time $(T=.28)$, and number of approaches $(T=.33)$. In other words, higher levels of grooming during treatment were associated with lower levels of fear in the subsequent test. In addition, freezing during treatment was correlated with more safety tests to the first approach $(T=.46)$ and more total safety tests $(T=.42)$. None of the other correlations were significant.

When each group was considered separately, somewhat different patterns of significance emerged for the different groups. For the FL group, there were no significant correlations. For the B group, grooming during treatment was significantly correlated with approach latency $(T=-.48)$, first grid time $(T=.53)$, total grid time $(T=.58)$, and number of approaches $(T=.56)$. Thus, grooming was associated with less fear for the B group. For the NB group, grooming 
was also significantly correlated with first grid time $(\mathrm{T}=.51)$, total grid time $(\mathrm{T}=.51)$, and safety and approach latencies $(\mathrm{Ts}=-.57)$. In addition, abortive avoidances during treatment were significantly correlated with safety and approach latency $(\mathrm{Ts}=.47)$ for the NB group. In other words, for the NB group, high numbers of abortive avoidances were significantly related to high levels of fear in the subsequent test. In addition, freezing was significantly correlated with safety tests to the first approach and total safety tests (Ts $=.55)$ for the NB group.

\section{Discussion}

The results of Experiment 2 indicate that all three flooding procedures were effective in reducing fear of the grids as indexed by a number of different measures. Both the FL and the NB treatments resulted in reductions in fear on seven of our nine measures of fear. The B treatment resulted in significant reductions on four of these measures and in marginally significant reductions on three others. In addition, the NB treatment was found to be superior to the $B$ treatment on six measures and superior to the FL treatment on four measures. Unlike Corriveau and Smith (1978), we did not find number of safety tests, either before the first approach or overall, to be sensitive measures of fear (see also Mineka et al., 1981).

As for Experiment 1, there are several possible explanations for the superiority of the NB procedure in reducing fear. First, if the immediate dumping procedure used during treatment acted as a mild punishment for the response, it is possible that the ledge became mildly aversive to these subjects (and/or lost its safety properties). From this perspective, the fact that the NB subjects approached the grids somewhat sooner and spent more time there may reflect this mild aversion to the ledge as much as reduced fear of the grids. Consistent with this hypothesis is the observation that three subjects in this group left the ledge voluntarily even before being dumped the first time. However, if this hypothesis were correct, then one would expect the number of abortive avoidances during treatment to have been negatively correlated with levels of fear during the subsequent fear observation period. The results showed, however, that the subjects that made more abortive avoidances during treatment (and hence had more opportunities to have their responses punished) also tended to show higher levels of fear in the subsequent fear test; that is, they stayed on the ledge more (correlations of abortive avoidance with: safety and approach latencies, Ts = .47 , first and total grid times, $\mathrm{Ts}=-.31$ ). A more definitive test of this hypothesis could also be made by developing an independent measure of fear of the grids that did not involve the possible confound of an aversion to the ledge.

A second explanation of the superiority of the NB procedure stems from the possibility that the NB sub- jects had a perception that they had control over the shocks. By being able to respond periodically during treatment, they may have shown a further reduction in fear that comes with extended avoidance training (e.g., Kamin, Brimer, \& Black, 1963; Mineka \& Gino, 1980; Starr \& Mineka, 1977). It was noted that subjects in this group did "barpress" the safety ledge during both the treatment and observation phases of the experiment. Unfortunately, this was not recorded systematically enough to provide any definitive support for the controllability notion.

The third possible explanation for the superiority of the NB procedure is simply that these subjects had received the most complete exposure to all parts of the nonreinforced CS (wall movement, the dumping process, and exposure to the grids) and to the removal of the response-CS termination contingency. This explanation is the most parsimonious of the three, but its viability will await the results of experiments designed to test more directly between the three alternatives.

Another interesting aspect of our results from Experiment 2 concerns the activity measurements during treatment, which in large part replicated those of Experiment 1 . The NB group again made the highest number of abortive avoidances, and the $B$ group made the fewest; the B group also showed the highest levels of freezing again, with the other two groups showing very low levels of freezing. The primary difference from Experiment 1 was that there were no group differences in rate of increase in grooming over time; all groups showed comparable increases in grooming.

The pattern of changes in activity over the 5-min treatment period also paralleled that seen in Experiment 1. Abortive avoidances decreased across trial blocks (except for the B group, which showed low levels throughout), and grooming increased substantially. For the one group (B) that showed substantial levels of freezing, there was first an increase and then a decrease, as in Experiment 1.

Also of interest in Experiment 2 were the significant relationships between some of the activity measures during treatment and levels of fear shown subsequently. In particular, when collapsing across the three treatment groups, grooming was found to be significantly related to low levels of fear, as indexed by five of the sensitive measures. The same relationships were also found to hold for the B and NB groups alone. For the NB group, abortive avoidances during treatment were significantly correlated with high levels of fear, as indexed by safety and approach latencies. Thus, it seems that for the B and NB groups, and for all three groups together, grooming during treatment is a good predictor of low levels of fear in the subsequent test as Baum (1969) might have predicted. It should be remembered, however, that, unlike Baum, we did not find grooming to be related to avoidance extinction in Experiment 1. 


\section{GENERAL DISCUSSION}

The results of Experiment 2 concerning the relative effectiveness of different flooding procedures in reducing fear parallel quite closely the results of Experiment 1 concerning the relative effectiveness of the same procedures in hastening avoidance extinction. ${ }^{2}$ In both experiments, all three flooding procedures were effective, but the NB procedure was more effective than the FL and $B$ procedures. There are several aspects of the NB procedure that may contribute to its superiority. It was the only treatment in which actual responses were allowed and, thus, in which the subjects were exposed to the removal of the response-CS termination contingency. In addition, it involved the most complete exposure to all parts of the nonreinforced CS, including not only wall movement but also the dumping procedure itself. Unfortunately, it is not possible to tease apart the relative contributions of these different aspects of the NB treatment because they are so intimately intertwined; that is, one cannot expose the subjects to the dumping process during treatment without allowing them to respond.

It is also of interest to note that the B procedure did not significantly enhance the effectiveness of the traditional flooding procedure, even though the subjects in the $B$ groups received exposure to an additional important part of the nonreinforced CS-wall movement. This failure to find differences between the effects of the FL and B treatments either in hastening AR extinction or in reducing fear occurred in spite of the fact that the two treatments did produce different patterns of activity during the treatment phase itself. In particular, subjects in the B groups of both experiments showed more freezing and less abortive avoidances than did subjects in the other two groups. Nonetheless, it is possible that longer exposure to the B treatment would enhance its efficacy relative to the traditional FL treatment. This possibility stems from the following observations. Freezing decreases and grooming increases across the 5-min treatment period. In addition, for the B group, freezing was associated with more avoidance responses in extinction (Experiment 1) and grooming was associated with less fear (Experiment 2). Thus, longer exposure to the $B$ treatment would have produced patterns of activity that would be predictive of fewer ARs and less fear. The same prediction does not necessarily hold for the FL treatment, because freezing and grooming were not predictive of avoidance responding or levels of fear for that group.

The parallel between the effects of the three treatments in hastening avoidance extinction and in reducing fear is of interest for two reasons. First, as mentioned in the introduction, Experiment 2 is the first to explore the effects of a variety of flooding techniques on reducing fear rather than simply on hastening avoidance extinction. Given the pattern of results that was obtained, it becomes plausible to argue that a reduction in fear of the grid floor may contribute to the effects of flooding in hastening avoidance extinction. Whether or not fear reduction is sufficient, however, is less clear, given the results of Mineka et al. (1981), who found that a small amount of flooding may be sufficient to reduce fear without affecting avoidance extinction.

The second reason why the parallel results of Experiments 1 and 2 are of interest stems from possible relevance to maximizing the effectiveness of flooding techniques in the treatment of human phobias. Our results suggest that variables which increase the effectiveness of such techniques in reducing behavioral avoidance may also increase their effectiveness in reducing other aspects of the fear response. The results also suggest that a new variant on flooding techniques might be quite effective. Traditionally, flooding techniques have involved prolonged exposure to the phobic object without allowing the client to make avoidance responses. In fact, Greist, Marks, Berlin, Gournay, and Noshirvani (1980) reported that advising clients to avoid their phobic object as much as possible may produce an increase in the intensity of their phobia. However, the present results suggest that limited exposure to the opportunity to make the avoidance response may be helpful if the response is now no longer successful in avoiding the feared situation. For example, as Shearman (1970) suggested, a client with a bus phobia could be taken into the bus and then allowed to escape, but only onto another bus.

A final comment on the implications of our results concerns their generality. As discussed in the introduction, the results of experiments on variations in flooding techniques for shuttleboxes seem to suggest that response-prevention per se may be important in enhancing their effectiveness, at least in facilitating avoidance response extinction. However, our results with a jump-up box avoidance response lead to an opposite conclusion. There are several possible explanations for this apparent discrepancy. First, as suggested by previous researchers, the superiority of the response-prevention procedure in the shuttlebox may stem from the possibility for competing responses (e.g., freezing) to be learned during traditional flooding treatments that involve responseprevention (cf. Berman \& Katzev, 1972; Coulter, Riccio, \& Page, 1969; Shipley, Mock, \& Levis, 1971). Extinction procedures in the shuttlebox do not generally involve anything that would tend to break up a competing response that was learned during the flooding treatment, and so the response-prevention procedures may be especially effective. In contrast, in the jump-up box, extinction generally starts with the subject's being placed on the ledge and then dumped onto the grid floor. This dumping procedure may serve to break up any competing response that has been learned during flooding (see Mineka \& Gino, 
$1979 \mathrm{~b}$, for a discussion). Thus, the opportunity for learning a competing response during responseprevention in a jump-up box is not as likely to contribute to the effectiveness of the procedure in hastening avoidance response extinction as it is in a shuttlebox. This possibility would be especially likely if it were found that, while response-prevention may be important for maximizing the efficacy of flooding in hastening shuttlebox avoidance response extinction, it may not be equally important in maximizing the efficacy of flooding in reducing independent measures of fear of a shuttlebox CS.

A second and related reason why responseprevention per se may have differential importance for hastening jump-up and shuttlebox avoidance extinction stems from consideration of the differences in the processes involved in the acquisition, maintenance, and extinction of two-way and one-way avoidance responses (cf. Bolles, 1970; Mineka, 1979). This, in turn, may mean that there are different learning processes mediating the effects of flooding in hastening extinction of the two different kinds of avoidance responses. For example, substantial fear reduction may be necessary to hasten jump-up avoidance extinction (e.g., Mineka et al., 1981), and, as seen in the results of Experiment 2, the NB procedure was highly effective in reducing fear. In contrast, fear reduction does not seem to be necessary for flooding to hasten two-way shuttlebox avoidance extinction (Mineka \& Gino, 1979a). Instead, responseprevention procedures may be ideal for disrupting shuttlebox responses because they maximize the opportunity for the learning of competing responses or because they disrupt any one of the delicate balance of factors involved in maintaining a shuttlebox response, without necessarily reducing fear. (See Mineka et al., 1981, for a further discussion of these issues.)

\section{REFERENCES}

BAUM, M. Extinction of an avoidance response following responseprevention: Some parametric investigations. Canadian Journal of Psychology, 1969, 23, 1-10.

BAUM, M. Extinction of avoidance responding through response prevention (flooding). Psychological Bulletin, 1970, 74, 276284.

BAUM, M. Extinction of avoidance behavior: Comparison of various flooding procedures in rats. Bulietin of the Psychonomic Society, 1973, 1, 22-24.

Berman, J. S., \& Katzev, R. D. Factors involved in the rapid elimination of avoidance behavior. Behaviour Research and Therapy, 1972, 10, 247-256.
Bolles, R. C. Species-specific defense reactions and avoidance learning. Psychological Review, 1970, 77, 32-48.

Corriveau, D. P., \& SmITh, N. F. Fear reduction and "safetytest" behavior following response-prevention: A multivariate analysis. Journal of Experimental Psychology: General, 1978, 107, 145-158.

Coulter, X., Riccio, D. C., \& PAge, H. A. Effects of blocking an instrumental avoidance response: Facilitated extinction but persistence of "fear." Journal of Comparative and Physiological Psychology, 1969, 68, 377-381.

Greist, J., Marks, I., Berlin, F., Gournay, K., \& Noshirvani, H. Avoidance versus confrontation of fear. Behavior Therapy, $1980,11,1-14$.

Kamin, L. J., Brimer, C. J., \& Black, A. H. Conditioned suppression as a monitor of fear of the CS in the course of avoidance training. Journal of Comparative and Physiological Psychology, 1963, 56, 497-501.

MineKA, S. The role of fear in theories of avoidance learning, flooding and extinction. Psychological Bulletin, 1979, 86, 9851010.

MineKa, S., \& Gino, A. Dissociative effects of different types and amounts of non-reinforced CS exposure on avoidance extinction and the CER. Learning and Motivation, 1979, 10, 141-160. (a)

MineKa, S., \& Gino, A. Some further tests of the brief confinement effect and the SSDR account of flooding. Learning and Motivation, 1979, 10, 98-115. (b)

Mineka, S., \& Gino, A. Dissociation between CER and extended avoidance performance. Learning and Motivation, 1980, 11, 476-502.

Mine Ka, S., Miller, S., Gino, A., \& Giencke, L. Dissociative effects of flooding on a multivariate assessment of fear reduction and on jump-up avoidance extinction. Learning and Motivation, $1981,12,435-461$.

Morokoff, P. J., \& Timberlake, W. Cue exposure and overt fear responses as determinants of extinction of avoidance in rats. Journal of Comparative and Physiological Psychology, 1971, 77, 432-438.

Polin, A. T. The effect of flooding and physical suppression as extinction techniques on an anxiety-motivated avoidance locomotor response. Journal of Psychology, 1959, 47, 253-255.

Shipley, R. H., Mock, L. A., \& Levis, D. J. Effects of several response prevention procedures on activity, avoidance responding, and conditioned fear in rats. Journal of Comparative and Physiological Psychology, 1971, 77, 256-270.

Stark, M. D., \& MineKA, S. Determinants of fear over the course of avoidance learning. Learning and Motivation, 1977, 8, 332-350.

TinsLey, H., \& WEISS, D. Interrater reliability and agreement of subjective judgments. Journal of Counseling Psychology, $1975,22,358-376$.

\section{NOTES}

1. Activity ratings were taken for only $\mathbf{4 0}$ of the subjects in Experiment 2 (10 from each group).

2. Although one or two of these measures of fear (jump latency and possibly first grid time) may in part reflect the strength of the avoidance response itself, the other measures seem to be quite independent of avoidance response strength.

(Manuscript received September 8, 1981; revision accepted for publication March 3, 1982.) 